\title{
conference abstracts
}

s10.m1.03 Potentialities of Grid Search Method. V.V. Chernyshev, Chemistry Dept., Moscow State University, 119899 Moscow, Russia.

Keywords: powder diffraction, advanced methods, structure determination.

A grid search method is one of the methods, which are now available to facilitate the crystal structure determination from powder data. The advantages of the methods are the simplicity of the algorithm and the easiness of its realization in a computer code. The method is based on two key procedures: systematic translation and rotation of a known, or approximately known, structural fragment within the asymmetric part of the unit cell and subsequent bond-restrained refinement. The flexible use of bond restraints allows the molecule to suffer considerable conformational alterations during the course of refinement, leading to the correct solution even if significant preferred orientation is present, or partly incorrect initial molecular model was used.

Potentialities of grid search method will be discussed on the basis of solutions of previously unknown molecular crystal structures of pharmaceutical compounds and anthracene-derived dyes, successfully solved from X-ray, synchrotron and neutron powder diffraction data. A new problem mentioned by some authors, when we can locate molecules in much bigger cells than we could refine by Rietveld method without constraints, will be discussed as well. s10.m1.04 Direct space method and high-resolution $\mathrm{X}$ ray powder diffraction: a modern approach to pharmaceutical crystal structure determination. J. Giovannini $^{1,2}$, M.-A. Perrin ${ }^{1}$, D. Louër ${ }^{2}$ and F. Leveiller ${ }^{1}$, ${ }^{I}$ Aventis Pharma, Vitry sur Seine, France $-{ }^{2}$ Laboratoire de Chimie du Solide et Inorganique Moléculaire, Université de Rennes 1, France.

Keywords: structure solution from powder data, drug compounds, simulated annealing.

Nowadays, for the determination of organic crystal structures, high-resolution X-ray powder diffraction (XRPD) data is the alternative to the single crystal approach. In the case of drug compounds for which it was difficult to obtain single crystals of appropriate quality, size and desired crystalline structure (metastable phases), it is even the only solution. Recent progresses have been made in the development of new computer programs ${ }^{1}$, which allow the determination of crystal structures directly from XRPD. In the present study PowderSolve $e^{2}$ was used. It is based on a Monte Carlo Simulated Annealing type approach which operates in direct space and uses solely the envelope of the powder pattern. A realistic and relevant structural solution depends on the quality of two major components, peak positions and intensities. The indexing of the powder pattern was performed using the Bragg Brentano geometry. To reduce the effect of transparency, a thin layer of powder was deposited on an oriented singlecrystal silicon wafer. Unit cell parameters were obtained using DICVOL91. To collect reliable intensities (minimisation of preferred orientation effects), the transmission geometry was used with a micronised powder introduced in a rotating Lindemann glass capillary with low absorption. Using a quantum molecular geometry, trial structures were generated by stochastic movements of rigid bodies within the unit cell. Powder patterns were simulated and then compared to the experimental data. The validity criteria of each model is defined by the $\mathrm{R}_{w p}$ profile factor. The final structure model has to fit both the physical sense of the molecular packing and the X-ray diffraction data satisfactorily. Crystal structures of drug compounds of increasing complexity have been solved with this approach:

$\mathrm{I}\left(C 2 / \mathrm{c}, \mathrm{V}=2512.5(3) \AA^{3}, 33\right.$ atoms (including $\mathrm{H}$ atoms), 2 torsions, $\left.\mathrm{R}_{w p}=7.72 \%\right)$, II $\left(P 2_{1}, \mathrm{~V}=1067.4(3)\right.$ $\AA^{3}, 54$ atoms, 5 torsions, $\left.\mathrm{R}_{w p}=15.34 \%\right)$, III $(P-1, \mathrm{~V}=$ 1068.2(5) $\AA^{3}, 54$ atoms, 5 torsions, $\left.\mathrm{R}_{w p}=12.85 \%\right)$. This methodology is now used routinely and has recently been applied to solve the structure of a compound exhibiting a very high configurational flexibility IV $\left(P 2_{1}, \mathrm{~V}=2266(7)\right.$ $\AA^{3}, 117$ atoms, 22 torsions, $\mathrm{R}_{w p}=11.71 \%$ ).
[1] Harris K. D. M. This Conference.

[2] Engel G. E., Wilke S., König O., Harris K. D. M., Leusen F. J. J., "PowderSolve - a complete package for crystal structure solution from powder diffraction patterns.", J. Appl. Cryst. (1999), 32, 1169-1179. 